CONFORMAL GEOMETRY AND DYNAMICS

An Electronic Journal of the American Mathematical Society

Volume 6, Pages 13-32 (January 24, 2002)

S $1088-4173(02) 00075-9$

\title{
ON THE FAILURE OF A GENERALIZED DENJOY-WOLFF THEOREM
}

\author{
PIETRO POGGI-CORRADINI
}

\begin{abstract}
The classical Denjoy-Wolff Theorem for the unit disk was generalized, in 1988, by Maurice Heins, to domains bounded by finitely many analytic Jordan curves. Heins asked whether such an extension is valid more generally. We show that it can actually fail for some domains. Specifically, we produce an automorphism $\phi$ on a planar domain $\Omega$, such that the iterates of $\phi$ converge to a unique Euclidean boundary point, but do not converge to a unique Martin point in the Martin compactification of $\Omega$. We then extend this example to a family of examples in the second part of this work. We thus consider the Martin boundary for domains whose complement is contained in a strip and generalize results of Benedicks and Ancona.
\end{abstract}

\section{INTRODUCTION}

Let $\phi$ be an analytic map of the disk unit disk $\mathbb{D}$ such that $\phi(\mathbb{D}) \subset \mathbb{D}$, we say $\phi$ is an endomorphism of $\mathbb{D}$ and write $\phi \in \operatorname{End}(\mathbb{D})$. If $z \in \partial \mathbb{D}$, then $\phi(z)$ and $\phi^{\prime}(z)$ are defined as non-tangential limits, no smoothness across the boundary is required. Aside for the case when $\phi$ is an elliptic automorphism, the following holds.

Theorem 1.1 (Denjoy-Wolff). There exists a unique point $\omega \in \overline{\mathbb{D}}$ such that the iterates $\phi_{n}$ converge to $\omega$ uniformly on compact subsets of the disk. Moreover, $\omega$ is the only fixed point of $\phi$ in $\overline{\mathbb{D}}$ satisfying $\left|\phi^{\prime}(\omega)\right| \leq 1$.

The special point $\omega$ is called the Denjoy-Wolff point of $\phi$. By Schwarz's Lemma, $\phi$ has at most one fixed point in $\mathbb{D}$ and if so, such fixed point must be the DenjoyWolff point.

Maurice Heins [He88] studied generalizations of Theorem 1.1 for analytic endomorphisms of general planar domains $\Omega$, and even Riemann surfaces, and succeeded in extending the Denjoy-Wolff Theorem to finitely connected domains with nice boundary. In [He41] Heins completely worked out the situation when the endomorphism has a fixed point in $\Omega$ and $\Omega$ is a planar domain whose complement contains more than 2 points (hyperbolic domain). If the endomorphism does not have a fixed point in $\Omega$, then the boundary of $\Omega$ comes into play. So let us review in more detail what happens when $\Omega=\mathbb{D}$. Say $\phi$ is an endomorphism of $\mathbb{D}$ with Denjoy-Wolff point at 1 . Since $\phi(1)=1$ and $\phi^{\prime}(1) \leq 1$, if $D$ is a horodisk at 1 , i.e., a disk contained in $\mathbb{D}$ which is tangent to $\partial \mathbb{D}$ at 1 , we must have $\phi(D) \subset D$, by Julia's Lemma. Note that these horodisks are the level sets of the Poisson kernel

Received by the editors April 6, 2001 and, in revised form, November 7, 2001.

2000 Mathematics Subject Classification. Primary 30D05, 31 A05.

The author was partially supported by NSF Grant DMS 97-06408. We thank Professor A. Ancona for very helpful conversations. 
at 1: $D=D(1, \lambda)=\left\{P_{1}(z)>\lambda\right\}$, for some $\lambda>0$, where $P_{1}(z)=\left(1-|z|^{2}\right) /|1-z|^{2}$. Therefore, we have

$$
P_{1}(\phi(z)) \geq P_{1}(z) .
$$

Moreover the converse also hold: if $\phi \in \operatorname{End}(\mathbb{D})$ and (1.1) holds, then either $\phi$ is the identity or $\phi_{n}$ tends to 1 uniformly on compact subsets of $\mathbb{D}$.

In order to generalize this to finitely connected domains, M. Heins considered the Martin compactification of $\Omega$. Let $\Omega$ be a domain whose boundary is regular for the Dirichlet problem. Then we can talk about Green's function $G(z, w)$. Fix a base point $z_{0} \in \Omega$ and to each point $w \in \Omega \backslash\left\{z_{0}\right\}$ assign the function $G(\cdot, w) / G\left(z_{0}, w\right)$. For every sequence $w_{n}$ tending to $\partial \Omega$, the corresponding sequence of functions forms a normal family on $\Omega$, and therefore one can extract a subsequence which converges to a positive harmonic function, uniformly on compact subsets of $\Omega$. We let $\partial_{M} \Omega$ be the family of all possible normal sublimits obtained this way: this is the Martin boundary of $\Omega$. The individual functions in $\partial_{M} \Omega$ are called Martin kernels. They are generalizations of Poisson kernels. For instance, if $\Omega=\mathbb{D}$ and $z_{0}=0$, then $\partial_{M} \Omega$ consists of the Poisson kernels $\left\{P_{\theta}(z)=\left(1-|z|^{2}\right) /\left|e^{i \theta}-z\right|^{2}\right\}$. One difference between the general case and the case of the unit disk is the existence of non-minimal Martin kernels. Recall Ba95] that a positive harmonic function $u$ on $\Omega$ is minimal if every harmonic function $v$ on $\Omega$ such that $0<v \leq u$ is of the form $v=c u$ for some constant $c>0$. In the case of the disk, the Poisson kernels are all minimal. On the other hand, in the general situation, a Martin kernel is always a weighted average of minimal Martin kernels. So minimal Martin kernels are the more appropriate generalization of Poisson kernels (there are also other reasons for this statement).

When $\Omega$ is a domain bounded by finitely many analytic Jordan curves, then the Martin boundary $\partial_{M} \Omega$ is in one-to-one correspondence with the regular Euclidean boundary $\partial \Omega$ and every Martin kernel is minimal. In this context Heins proves,

Theorem 1.2 ([He88]). Suppose $\Omega$ is a domain bounded by finitely many analytic Jordan curves. If $\phi \in \operatorname{End}(\Omega)$ does not have a fixed point in $\Omega$, then there exists a point $q \in \partial \Omega$ such that for every $z \in \Omega, \phi_{n}(z)$ tends to $q$ and if $U_{q}$ is the Martin kernel corresponding to $q$, then

$$
U_{q}(\phi(z))>U_{q}(z) \quad \text { for all } z \in \Omega .
$$

Equation (1.2) should be compared to (1.1) above. It turns out in this case that the sequence $\phi_{n}(z)$ also tends to $U_{q}$ in the Martin topology, namely the ratios

$$
\frac{G\left(\cdot, \phi_{n}(z)\right)}{G\left(z_{0}, \phi_{n}(z)\right)}
$$

tend to $U_{q}(\cdot)$, uniformly on compact subsets of $\Omega$.

In the same paper, Heins states: It is natural to ask to what extent the results of Denjoy and Wolff remain valid when $\mathbb{D}$ is replaced by a Riemann surface $S$ admitting non-constant positive harmonic functions and $\phi$ by a holomorphic map of $S$ into itself. The general problem does not appear to be simple.

This question was related to us in a more precise form by Alex Eremenko: Suppose $\Omega$ is a planar domain whose boundary is regular for the Dirichlet problem, $\phi \in \operatorname{End}(\Omega)$ has no fixed points in $\Omega$, and there is a Euclidean boundary point $\zeta \in \partial \Omega$ such that for each $z \in \Omega, \phi_{n}(z)$ tends to $\zeta$, as $n \rightarrow \infty$. Does there exist a Martin kernel $H$ such that $\phi_{n}(z)$ tends to $H$ in the Martin compactification, as 
$n \rightarrow \infty$ ? and such that $H(\phi(z)) \geq H(z)$, for all $z \in \Omega$ ? The problem here is that in general the Euclidean boundary point $\zeta$ corresponds to multiple Martin boundary points.

In this note we show that the answer to this question is no. We are indebted to Professor Alano Ancona for pointing us in the right direction and for bringing to our attention the beautiful boundary Harnack principle of Section 7 in An84. In Section 2 we describe the counterexample and offer a proof based on an estimate of Carleson. In Section 3 we relate Ancona's approach. Finally, in Sections 4 and 5 we generalize the counterexample to translation-invariant domains whose complement is contained in a strip, and in doing so, we prove some results of independent interest about arbitrary domains for which the complement is contained in a strip. We tried to include a few pictures, however this paper is best read by drawing the relevant pictures at each step, and we encourage the reader to do so. Section 6 contains further results and some open questions.

\section{Description of the COUnterexample}

Let $E=\bigcup_{n \in \mathbb{Z}}[n+1 / 2-\epsilon, n+1 / 2+\epsilon]$, with $\epsilon<1 / 2$. Then the domain $\Omega=\mathbb{C} \backslash E$ is translation-invariant, i.e., $\Omega+1=\Omega$. Also, every point on $\partial \Omega$ is regular for the Dirichlet problem, including infinity, as can be seen using Wiener's criterion (see Figure 1).

The Martin boundary of $\Omega$ with base point at the origin, is described below. For proofs of these facts see [An79] or [Be80].

a) At every $\zeta \in(n+1 / 2-\epsilon, n+1 / 2+\epsilon)$ there are two minimal Martin kernels (one for each side).

b) At the end-points $\zeta=n+1 / 2 \pm \epsilon$ there is only one minimal Martin kernel.

c) At $\infty$, the boundary is thick enough to split as in case a); so there are two minimal Martin kernels, namely:

- $H^{+}(z)$ is the kernel that one obtains by letting a sequence $z_{n}$ tend to infinity in a sector of opening strictly less than $\pi$ symmetric about the positive imaginary axis. It turns out that $H^{+}$is bounded on the lower half-plane $\{\operatorname{Im} z \leq 0\}$.

- On the other hand, $H^{-}(z)=H^{+}(\bar{z})$ is bounded on the upper half-plane, and corresponds to sequences tending to infinity (non-tangentially) in the lower half-plane.

Note that the Euclidean boundary point at $\infty$ is different from those in case a) because it also supports non-minimal kernels. Namely, every convex combination $H(z)=\alpha H^{+}(z)+\beta H^{-}(z)$, with $\alpha+\beta=1$ and $\alpha, \beta \geq 0$, belongs to the Martin boundary at infinity. (This follows from the connectedness of the closure of the sets $\{z \in \Omega:|z| \geq R\}$ in the Martin topology.)

The automorphism in this case is the translation $\phi(z)=z+1$, and for each $z \in \Omega$ the sequence $\phi_{n}(z)=z+n$ converges to $\infty \in \partial \Omega$. The generalized Denjoy-Wolff Theorem would then require that $z+n$ tend to a unique Martin point at infinity. It turns out that this is not the case.

In fact, given $x \in \mathbb{R} \backslash E$, the sequence $x+n$ tends to the midpoint $(1 / 2) H^{+}+$ $(1 / 2) H^{-}$as $n \rightarrow \infty$. To see this, consider the normalized Green functions

$$
g_{n}(z)=\frac{G(z, x+n)}{G(0, x+n)} .
$$




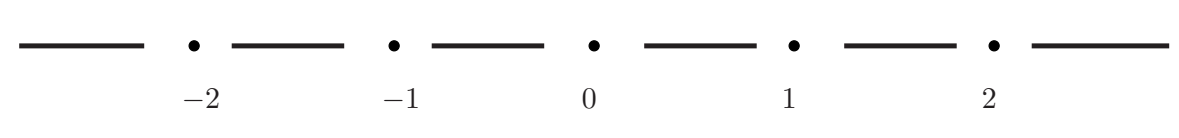

Figure 1. Counterexample

By symmetry, $g_{n}(z)=g_{n}(\bar{z})$. So every normal sublimit as $n \rightarrow \infty$ is a Martin kernel at infinity which must also be symmetric about $\mathbb{R}$, hence it can only be the midpoint $(1 / 2) H^{+}+(1 / 2) H^{-}$.

Suppose now that $\operatorname{Im} w>0$. We claim that the sequence $w+n$ does not tend to the midpoint $(1 / 2) H^{+}+(1 / 2) H^{-}$in the Martin topology, as $n \rightarrow \infty$. To this end we will show that the function

$$
v_{n}(z)=\frac{G(z, w+n)}{G(0, w+n)}-\frac{G(\bar{z}, w+n)}{G(0, w+n)}
$$

does not tend to zero as $n \rightarrow \infty$.

Let $\mathbb{H}=\{z \in \mathbb{C}: \operatorname{Im} z>0\}$. Note that the function $v_{n}$ is harmonic in $\mathbb{H} \backslash\{w+n\}$, vanishes on $\mathbb{R} \cup\{\infty\}$, and has a positive logarithmic pole at $w+n$. Thus $v_{n}$ is positive on $\mathbb{H} \backslash\{w+n\}$. We let $B_{n}=\{z \in \Omega: G(z, w+n) \geq 1 / \delta\}$, with $\delta$ small enough so that $B_{n} \cap \mathbb{R}=\emptyset$. Then by invariance, $B_{n}=B+n$ (where $B=B_{0}$ ), and by the Maximum Principle, $G(z, w+n)=(1 / \delta) \omega\left(z, B_{n}, \Omega\right)$ for all $z \in \Omega \backslash B_{n}$. (Here $\omega$ denotes harmonic measure.) Thus for $z \in \Omega \backslash B_{n}$,

$$
v_{n}(z)=\frac{\omega\left(z, B_{n}, \Omega\right)-\omega\left(\bar{z}, B_{n}, \Omega\right)}{\omega\left(0, B_{n}, \Omega\right)} .
$$

The numerator is a harmonic function on $\mathbb{H} \backslash B_{n}$, which vanishes on $\mathbb{R} \cup\{\infty\}$, and which, on $\partial B_{n}$, is greater than the constant

$$
C:=1-\max _{\bar{z} \in B_{n}} \omega\left(z, B_{n}, \Omega\right)=1-\max _{\bar{z} \in B} \omega(z, B, \Omega)>0 .
$$

Thus for $z \in \mathbb{H} \backslash B_{n}$,

$$
v_{n}(z) \geq C \frac{\omega\left(z, B_{n}, \mathbb{H}\right)}{\omega\left(0, B_{n}, \Omega\right)}
$$

Now we estimate the rates of decay of numerator and denominator.

We start with the numerator. Assume that $z$ is fixed in $\mathbb{H}$. Since $B$ has nonempty interior in $\mathbb{H}$, we can find a closed disk such that $\Delta \subset B$. Thinking of $\Delta$ as a hyperbolic disk with center $a$ and radius $r$, we apply the conformal map $(\zeta-(a+n)) /(\zeta-(\bar{a}+n))$ which maps $\mathbb{H}$ to $\mathbb{D}$ and maps $\Delta+n$ to a disk centered 
at 0 with Euclidean radius $r_{0}<1$. Then

$$
\begin{aligned}
\omega(z, B+n, \mathbb{H}) & \geq \omega(z, \Delta+n, \mathbb{H})=\frac{1}{\log r_{0}} \log \frac{|z-(\bar{a}+n)|}{|z-(a+n)|} \\
& =\frac{1}{2 \log r_{0}} \log \frac{|z-\bar{a}|^{2}+n^{2}-2 n \operatorname{Re}(z-\bar{a})}{|z-a|^{2}+n^{2}-2 n \operatorname{Re}(z-a)} \\
& =\frac{1}{2 \log r_{0}} \log \left(1+\frac{|z-\bar{a}|^{2}-|z-a|^{2}}{|z-a|^{2}+n^{2}-2 n \operatorname{Re}(z-a)}\right) \\
& \geq \frac{M}{n^{2}}
\end{aligned}
$$

for $n$ large and for some constant $M>0$ independent of $n$.

For the denominator, we recall an estimate due to Carleson:

$$
\omega(0,[n+1 / 2-\epsilon, n+1 / 2+\epsilon], \Omega) \leq \frac{A}{n^{2}}
$$

where $A>0$ is a constant independent of $n$. (See Ca82 p. 30, Ko88 Chap. VIII for an exposition, and [So94 for a shorter proof.)

By the maximum principle and invariance,

$$
\begin{aligned}
\omega(0, & {[n+1 / 2-\epsilon, n+1 / 2+\epsilon], \Omega) } \\
& \geq \omega\left(0, B_{n}, \Omega\right) \min _{\zeta \in B_{n}} \omega(\zeta,[n+1 / 2-\epsilon, n+1 / 2+\epsilon], \Omega) \\
& =\omega\left(0, B_{n}, \Omega\right) \min _{\zeta \in B} \omega(\zeta,[1 / 2-\epsilon, 1 / 2+\epsilon], \Omega) .
\end{aligned}
$$

So

$$
\omega\left(0, B_{n}, \Omega\right) \leq \frac{A^{\prime}}{n^{2}}
$$

In conclusion, we find that

$$
v_{n}(z) \geq C \frac{M / n^{2}}{A^{\prime} / n^{2}}=C_{0}
$$

where $C_{0}>0$ is a constant independent of $n$, and this proves our claim.

\section{ANCONA'S APPROACH}

The arguments in this section were kindly suggested to us by Professor Alano Ancona. The same conclusions as in Section 2 can be obtained using Ancona's version of the Boundary Harnack Principle for Denjoy domains [An84. Recall that Denjoy domains are planar domains whose complement is contained in the real axis. Ancona's result is actually more general and works for domains whose complement is contained in the graph of a Lipschitz function defined on $\mathbb{R}^{n}$.

Let $\Omega$ be a domain in $\mathbb{R}^{3}\left(\mathbb{R}^{n}, n \geq 2\right.$, is similar) whose complement is contained in the graph $\Sigma=\left\{(x, y, z) \in \mathbb{R}^{3}: z=F(x, y)\right\}$ of a Lip1 function $F$ (i.e., $\left.\left|F(x, y)-F\left(x^{\prime}, y^{\prime}\right)\right| \leq L \sqrt{\left(x-x^{\prime}\right)^{2}+\left(y-y^{\prime}\right)^{2}}\right)$. The Lipschitz constant $L$ is assumed to be fixed. Let $T(r)$ be the circular cylinder about the $z$-axis, symmetric about the $(x, y)$-plane, with radius $r$ and height $h=10 L r$. Set $T(O, r)=O+T(r)$ for a point $O$ on $\Sigma$. Also let $\overline{O A_{1}}$ be the vertical segment in $T(O, r)$ above $O$, and $\overline{O A_{2}}$ the vertical segment below $O$ in $T(O, r)$. This is Théoreme 7.3 of An84. 
Theorem 3.1 (Ancona's Boundary Harnack Principle for Denjoy domains). Suppose $u$ and $v$ are harmonic on $T(O, 2 r) \cap \Omega,>0$ there, and $=0$ on $T(O, 2 r) \backslash \Omega$. Then for all $P \in T(O, r) \cap \Omega$,

$$
\frac{u(P)}{v(P)} \leq C \max _{j=1,2} \frac{u\left(A_{j}\right)}{v\left(A_{j}\right)}
$$

for some constant $C$ which depends only on $L$.

Let $\Omega$ be the domain $\mathbb{C} \backslash E$ defined in Section 2, and recall that the main point in using Carleson's estimate was to show that

$$
\omega\left(0, B_{n}, \Omega\right) \leq \frac{A^{\prime}}{n^{2}}
$$

where $B_{n}=\{z \in \Omega: G(z, w+n) \geq 1 / \delta\}$ and $\delta$ is small enough. Again $\omega\left(0, B_{n}, \Omega\right)=$ $\delta G(0, w+n)$, so this time we focus on $G(0, w+n)$ instead.

By Harnack's inequality, we can assume that $w=0$. Then, we apply Theorem 3.1 to the square $Q(0, n)=\{|\operatorname{Re} z|<n,|\operatorname{Im} z|<n\}$. That is to say, we choose $L$ and $r$ above so that $T(O, 2 r)$ is exactly equal to $Q(0, n)$ in this context (say $L=1 / 5$ and $r=n)$. Also we let $u(z)=G(z, n)$ and $v(z)=G(z, n i)+G(z,-n i)$. Note that in this case, $A_{1}=n i / 2, A_{2}=-n i / 2$. Hence, we obtain that

$$
\begin{aligned}
\frac{G(0, n)}{2 G(0, n i)} & \leq C \max \left\{\frac{G(n i / 2, n)}{G(n i / 2, n i)+G(n i / 2,-n i)}, \frac{G(-n i / 2, n)}{G(-n i / 2, n i)+G(-n i / 2,-n i)}\right\} \\
& \leq C \frac{G(n i / 2, n)}{G(n i / 2, n i)}
\end{aligned}
$$

where we used the symmetry of $\Omega$ with respect to the real axis, and positivity of Green functions (see Figure 21). Note that

$$
G(n i / 2, n i) \geq G_{\mathbb{H}}(n i / 2, n i)=\log 3
$$

where $G_{\mathbb{H}}$ is the Green function for the upper half-plane.

Finally, by Harnack and invariance, $G(n i / 2, n) \leq C_{2} G(n+n i, n)=C_{2} G(n i, 0)$, where $C_{2}$ is the Harnack constant for the set $\{i / 2,1+i\}$ in the upper half-plane. Thus

$$
G(0, n) \leq C[G(0, n i)]^{2}
$$

for some constant $C$ independent of $n$.

Now recall that every positive harmonic function vanishing on the boundary of $\Omega$ is a linear combination of the two Martin kernels, $\mathrm{H}^{+}$and $\mathrm{H}^{-}$, introduced at the beginning of Section 2 Hence, by Case 2 in item (III) of Theorem 3 of [Be80, we have that

$$
H^{+}(z) \geq \operatorname{Im} z
$$

for all $z \in \mathbb{H}$. Applying Theorem 3.1 again to the square $Q(0, n)$ as above, but this time with the functions $G(z, n i)$ and $H^{+}(z)+H^{-}(z)$, we obtain:

$$
\frac{G(0, n i)}{H^{+}(0)} \leq 2 C \max \left\{\frac{G(n i / 2, n i)}{H^{+}(n i / 2)+H^{-}(n i / 2)}, \frac{G(-n i / 2, n i)}{H^{+}(-n i / 2)+H^{-}(-n i / 2)}\right\} .
$$

Note that $H^{+}(0)=1, H^{+}(z)=H^{-}(\bar{z})$. Also

$$
G(n i / 2, n i) \leq C_{1}
$$




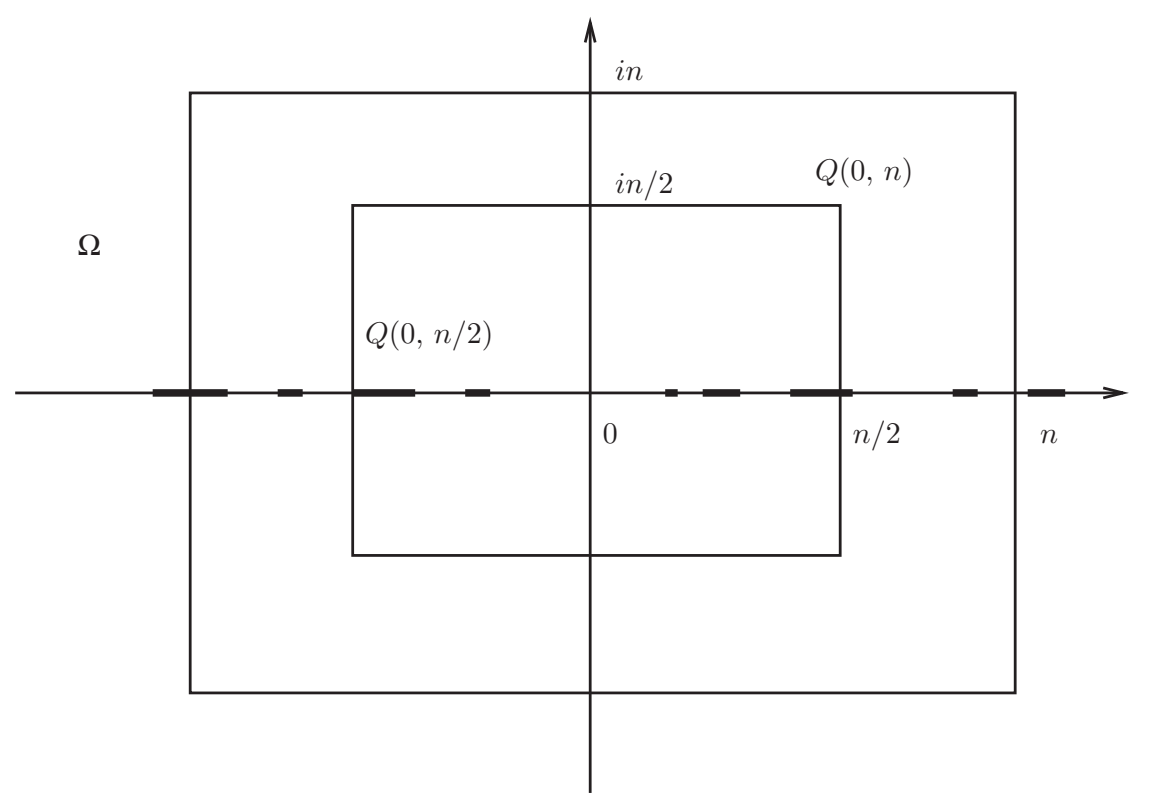

FiguRE 2. Boundary Harnack for Denjoy domains

with $C_{1}$ independent of $n$. To see this, apply the map $z / n$ and then the Möbius transformation $(z-i) /(z+i)$ followed by another dilation $z / 2$, so that $n i / 2$ and $n i$ are mapped to two points $a$ and $b$ in the disk of radius $1 / 2$ centered at the origin and $\Omega$ is mapped to a domain $\tilde{\Omega}$ whose complement is within one unit of $a$ and $b$. By definition of Green's function and conformal invariance:

$$
G(n i / 2, n i)=\tilde{G}(a, b)=\log \frac{1}{|a-b|}+U(a)
$$

where $\tilde{G}$ is the Green function for $\tilde{\Omega}$ and $U$ is the solution to the Dirichlet problem on $\tilde{\Omega}$ with data $\log |\zeta-b|$. Thus $U(a) \leq 0$, and $C_{1}=\log 1 /|a-b|$.

Also, $G(-n i / 2, n i) \leq G(n i / 2, n i)$, as we can see by applying the maximum principle to the function $G(z, n i)-G(\bar{z}, n i)$ on the upper half-plane. In conclusion, we have

$$
G(0, n i) \leq \frac{C}{H^{+}(n i / 2)} \leq \frac{2 C}{n}
$$

and therefore, $G(0, n) \leq C / n^{2}$, for some other constant $C>0$.

\section{Domains Whose COMPlement is CONTAIned In A STRIP}

As we mentioned before, Ancona's Boundary Harnack Principle is quite general, so one could think of generalizing the example of Section 2. For instance, consider a domain $\Omega$ which is translation-invariant $(\Omega+1=\Omega)$, and whose complement is contained in the graph of a Lipschitz function defined on $\mathbb{R}$. Then, the same conclusions should follow. Namely, the Martin boundary at infinity should be equal to the set $\left[\mathrm{H}^{+}, \mathrm{H}^{-}\right]$of convex combinations of $\mathrm{H}^{+}$and $\mathrm{H}^{-}$, where $\mathrm{H}^{+}$is a minimal Martin kernel bounded on the lower half-plane and $H^{-}$is a minimal Martin kernel bounded on the upper half-plane. Moreover, the normalized Green 
functions $G(z, w+n) / G\left(z_{0}, w+n\right)$ should, for different choices of $w$, tend to different convex combinations of $H^{+}$and $H^{-}$.

Instead of writing the details of this family of examples, which can be obtained using [An79] and [An84, we will describe a more general situation, which we think is of independent interest. Suppose, in fact, that $\Omega+1=\Omega$, and that the complement of $\Omega$ is contained in a strip (all our domains are always regular for the Dirichlet problem). Then, similar conclusions can be reached. In particular, we describe the Martin boundary at infinity in this case. To this end, we actually consider an even more general situation where $\Omega$ is a domain whose complement is contained in a strip, but $\Omega$ is not necessarily translation-invariant.

Recall that a positive harmonic function is said to be minimal if whenever $v$ is a harmonic function such that $0<v \leq u$, then $v \equiv c u$ for some constant $c>0$. Also $u$ is said to be of finite order if

$$
\max _{|z|=r} u(z) \leq C r^{p}
$$

for some $p>0$. Finally, let

$$
\beta(u)=\limsup _{r \rightarrow \infty}\left\{\omega(i,|z|=r, \Omega) \max _{|z|=r} u(z)\right\} .
$$

Theorem 4.1. Suppose $\Omega$ is a domain with $E=\mathbb{C} \backslash \Omega \subset\{-1 / 2<\operatorname{Im} z<0\}$, such that the logarithmic capacity $\operatorname{Cap}(E)$ of $E$ is strictly positive, and such that $\partial \Omega$ is regular for the Dirichlet problem. Let $\mathcal{P}$ be the family of positive harmonic functions on $\Omega$ vanishing on $\partial \Omega$ and $\mathcal{F}$ the subfamily of functions of finite order.

(i) Suppose $u \in \mathcal{P}$. Then $u \in \mathcal{F}$ if and only if $\beta(u)<\infty$. Moreover, in this case

$$
\max _{|z|=r} u(z) \leq C r
$$

(ii) There are either one or two linearly independent, minimal positive harmonic functions, vanishing on $\partial \Omega$, equal to 1 at $i$, and of finite order (briefly put $\operatorname{dim} \mathcal{F}=1$ or 2$)$.

(iii) Let $\omega^{+}(z)=\omega(z, \mathbb{R}+i, \Omega)$ and $\omega^{-}(z)=\omega(z, \mathbb{R}-i, \Omega)$. If the following thickness condition is satisfied,

$$
\sup _{x \in \mathbb{R}} \max \left\{\omega^{+}(x), \omega^{-}(x)\right\}<1
$$

then $\operatorname{dim} \mathcal{F}=2$.

(iv) Suppose that $\Omega+1=\Omega$ (translation-invariance). Then $\mathcal{P}=\mathcal{F}$.

Remark 4.2. M. Sodin [So94] considered a thickness condition which is sufficient for (4.2): for every $x \in \mathbb{R}$ and for some $\delta>0$ :

$$
\operatorname{Cap}\left[(\mathbb{C} \backslash \Omega) \cap Q_{x}\right] \geq \delta
$$

where $Q_{x}$ is the square of side 1 centered at $x$. In particular, this holds for translation-invariant domains. Sodin also proves a version of Carleson's estimate (2.1) for this type of domain.

Question 4.3. Is it true that $\sup _{x \in \mathbb{R}} \omega^{+}(x)<1$ if and only if $\sup _{x \in \mathbb{R}} \omega^{-}(x)<1$ ? What is the relationship between (4.2) and other thickness conditions such as (4.3), or other geometric conditions similar to the one assumed in Theorem 1 of [Ga89]? 


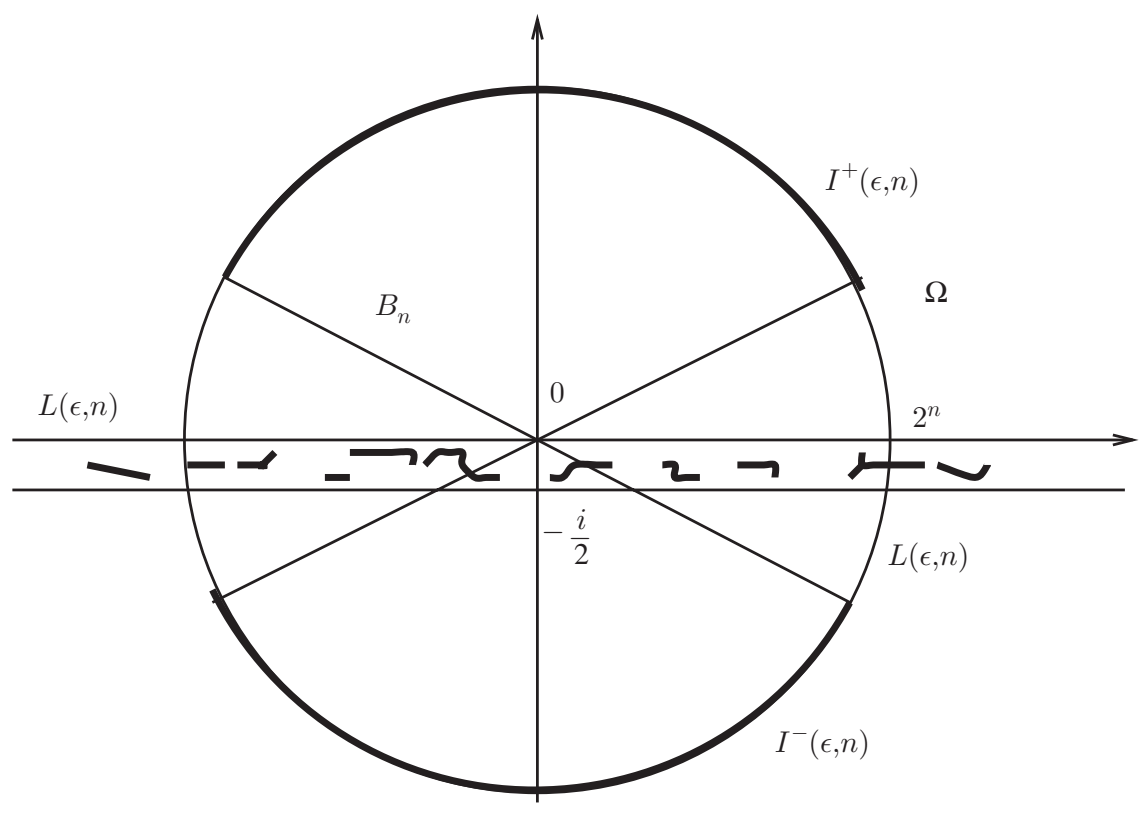

FiguRE 3. Decomposition

Question 4.4. Is it the case that given an arbitrary unbounded planar domain $\Omega$ there always is a positive harmonic function $u$ on $\Omega$ for which $\beta(u)<\infty$ ? If not, we ask the same question, and also whether Theorem4.1 (i) still holds, when $\omega(i,|z|=r, \Omega) \leq C r^{-q}$, for some $q>0$, as $r$ tends to infinity.

Since the proof of Theorem 4.1 is quite lengthy we will split it into subsections.

4.1. Harmonic measure gives a natural rate of growth. Here we prove Theorem 4.1 (i). We start by showing the sufficiency part. Note that

$$
\omega(i,|z|=r, \Omega) \geq \omega(i,|z|=r, \operatorname{Im} z>1 / 4) \geq \frac{C}{r} .
$$

So $\beta(u)<\infty$ implies that

$$
\max _{|z|=r} u(z) \leq C r
$$

and $u$ is of finite order.

The necessity part of (i) requires more work. If $\beta(u)=\infty$, we show that $u$ is not of finite order. We follow an argument similar to that in Lemma 3.5 of [ADP-C].

For $n=1,2,3, \ldots$ we set $T_{n}=\Omega \cap\left\{|z|=2^{n}\right\}, B_{n}=\Omega \cap\left\{|z| \leq 2^{n}\right\}$, and for $\epsilon>0$ we set $L(\epsilon, n)=T_{n} \cap\{|\arg z|<\epsilon,|\arg z-\pi|<\epsilon\}$, and $I(\epsilon, n)=T_{n} \backslash L(\epsilon, n)$. Also let $I^{+}(\epsilon, n)\left(I^{-}(\epsilon, n)\right)$ be the intersection of $I(\epsilon, n)$ with the upper (lower) half-plane. (See Figure 3.) Let $\mathcal{S}$ be the family of all domains $\Omega$ with $E=\mathbb{C} \backslash \Omega \subset$ $\{-1 / 2<\operatorname{Im} z<0\}$, Cap $E \neq 0$, and $\partial \Omega$ regular for the Dirichlet problem. Let $G_{n}(z, i)$ be the Green function for $B_{n}$ with pole at $i$.

Notations 4.5. We say that " $A \simeq B$ with constant $C$ ", meaning that $A / C \leq B \leq$ $C A$, for some $C>1$. 
Claim 4.6. With the notations above,

$$
\omega\left(i, I^{+}(\pi / 4, n), B_{n}\right) \simeq \omega\left(i, T_{n}, B_{n}\right) \simeq G_{n}\left(2^{n-1} i, i\right)
$$

with constants $C$ independent of $n \geq 3$ and $\Omega \in \mathcal{S}$.

Corollary 4.7. With the notations above,

$$
\omega\left(i, I(\epsilon, n), B_{n}\right) \simeq \omega\left(i, I(\epsilon, n+1), B_{n+1}\right)
$$

with a constant $\tau_{0}$ independent of $n \geq 3$ and $0 \leq \epsilon \leq \pi / 4$. (Note that in the case $\epsilon=0, I(0, n)=T_{n}$.)

Corollary 4.8. With the notations above,

$$
\omega\left(-i, I^{-}(\pi / 4, n), B_{n}\right) \simeq \omega\left(-i, T_{n}, B_{n}\right) .
$$

Claim 4.9. For any $\delta>0$, there exists an $\epsilon>0$ so that, for any domain $\Omega \in \mathcal{S}$, if $L(\epsilon, n), I(\epsilon, n), B_{n}$ are defined as above, and $n=1,2,3, \ldots$, then

$$
\omega\left(i, L(\epsilon, n), B_{n}\right) \leq \delta \omega\left(i, I(\epsilon, n), B_{n}\right) .
$$

We use these claims to prove the necessity part of Theorem 4.1 (i). Their proof is postponed for the moment.

By Claim 4.9, we can choose $\epsilon$ so small that $\omega\left(i, I(\epsilon, n), B_{n}\right) \geq(1 / 2) \omega\left(i, T_{n}, B_{n}\right)$, for $n=1,2,3, \ldots$. There is $n_{0}=n_{0}(\epsilon)$ so that $I(\epsilon, n)$ does not intersect the strip $\{|\operatorname{Im} z| \leq 1\}$ for all $n \geq n_{0}$. Then, by Claim 4.6] and Corollary 4.8 for $n \geq n_{0}$, given $u \in \mathcal{P}$,

$$
\begin{aligned}
u( \pm i) & \geq \omega\left( \pm i, I^{ \pm}(\epsilon, n), \Omega\right) \min _{z \in I^{ \pm}(\epsilon, n)} u(z) \\
& \geq B \omega\left( \pm i, T_{n}, B_{n}\right) u\left( \pm 2^{n} i\right) \\
& \geq A B \omega\left(i, T_{n}, B_{n}\right) u\left( \pm 2^{n} i\right)
\end{aligned}
$$

where $B=B(\epsilon)$ is the Harnack constant of $I^{ \pm}(\epsilon, n)$ which can be taken to be independent of $\Omega \in \mathcal{S}$, and $A=A(\Omega)$ is the Harnack constant of $\{ \pm i\}$ in the domain $\Omega$. We will see below, in Lemma 4.10, that the dependency on $\Omega \in \mathcal{S}$ can be dropped, but we don't need this here. In particular, 4.9 implies that when $\beta(u)=\infty, u$ must grow rapidly away from the sequence $\left\{ \pm 2^{n} i\right\}$, in view of (4.4).

Set $M_{n}=\max _{T_{n}} u$ and let $S>1$ be arbitrarily given. Note that

$$
\sup _{|z| \leq 1 / 2} \omega\left(z,\left\{e^{i \theta}:|\theta| \leq \epsilon\right\}, \mathbb{D}\right) \longrightarrow 0
$$

as $\epsilon$ tends to zero. So, by harmonic majorization, we can choose $\epsilon$ above so that

$$
\sup _{z \in T_{n}} \omega\left(z, L(\epsilon, n+1), B_{n+1}\right) \leq \frac{1}{2 \tau_{0} S}
$$

where $\tau_{0}$ is the constant of Corollary 4.7 Using the maximum principle for $z \in B_{n}$, we obtain

$$
u(z) \leq M_{n+1} \omega\left(z, L(\epsilon, n+1), B_{n+1}\right)+\max _{\zeta \in I(\epsilon, n+1)} u(\zeta) .
$$

Thus, letting $z$ range in $T_{n}$,

$$
M_{n} \leq M_{n+1} \frac{1}{2 \tau_{0} S}+\max _{\zeta \in I(\epsilon, n+1)} u(\zeta) .
$$


Note that by Corollary 4.7 (with $\epsilon=0$ ) and (4.9),

$$
\max _{\zeta \in I(\epsilon, n+1)} u(\zeta) \leq \frac{A}{\omega\left(i, T_{n}, B_{n}\right)}
$$

for $n \geq n_{0}(\epsilon)$ and some constant $A=A(\epsilon, \Omega, u)>0$.

Thus, multiplying both sides of (4.11) by $\omega\left(i, T_{n}, B_{n}\right)$, and using Corollary 4.7 again,

$$
M_{n} \omega\left(i, T_{n}, B_{n}\right) \leq M_{n+1} \omega\left(i, T_{n+1}, B_{n+1}\right) \frac{1}{2 S}+A .
$$

Iterating this inequality, for every integer $k \geq 1$,

$$
M_{n} \omega\left(i, T_{n}, B_{n}\right) \leq \frac{1}{(2 S)^{k}} M_{n+k} \omega\left(i, T_{n+k}, B_{n+k}\right)+A \sum_{j=0}^{k-1} \frac{1}{(2 S)^{j}} .
$$

Since $\beta(u)=\infty$, we can choose $n_{1}>n_{0}$ large enough so that

$$
M_{n_{1}} \omega\left(i, T_{n_{1}}, B_{n_{1}}\right)>\frac{A}{1-1 /(2 S)}+1 .
$$

Letting $n=n_{1}$ in (4.13) we obtain for every $k \geq 1$,

$$
M_{n_{1}+k} \omega\left(i, T_{n_{1}+k}, B_{n_{1}+k}\right) \geq(2 S)^{k} .
$$

Hence, for $n$ greater than some $n_{2}>n_{1}$,

$$
M_{n} \omega\left(i, T_{n}, B_{n}\right) \geq S^{n} .
$$

Since $S$ is arbitrary, $u$ is of infinite order and $u \notin \mathcal{F}$, i.e. Theorem 4.1 (i) is proved.

Proof of Corollary 4.7. Note that 4.6 follows from (4.5) because,

$$
\begin{aligned}
\omega\left(i, I(\epsilon, n+1), B_{n+1}\right) & \geq \omega\left(i, I(\pi / 4, n), B_{n+1}\right) \min _{\zeta \in I(\pi / 4, n)} \omega\left(\zeta, I(\epsilon, n+1), B_{n+1}\right) \\
& \geq \omega\left(i, I(\pi / 4, n), B_{n}\right) C_{0} \\
& \geq \omega\left(i, I(\epsilon, n), B_{n}\right)\left(C_{0} / C\right)
\end{aligned}
$$

where $C_{0}=\min _{\zeta \in I(\pi / 4,0)} \omega\left(\zeta, I(\pi / 4,1), Q_{1}\right)$ in the domain $Q_{1}=\{|z|<2, \operatorname{Im} z>1 / 2\}$, and where the last inequality follows from (4.5).

Proof of Corollary 4.8. Applying Claim 4.6 to $\tilde{\Omega}=\{z-i / 2: \bar{z} \in \Omega\}$ we find that, if $\tilde{B}_{n}$ are balls centered at $-i / 2$ of radius $2^{n}$, with the obvious changes of notation,

$$
\omega\left(-i, \tilde{I}^{-}(\pi / 4, n), \tilde{B}_{n}\right) \simeq \omega\left(-i, \tilde{T}_{n}, \tilde{B}_{n}\right)
$$

However, since $\tilde{I}^{-}(\pi / 4, n-1) \subset B_{n}$ for $n=2,3, \ldots$,

$$
\begin{aligned}
\omega(-i & \left.I^{-}(\pi / 4, n), B_{n}\right) \\
& \geq \omega\left(-i, \tilde{I}^{-}(\pi / 4, n-1), \tilde{B}_{n-1}\right) \min _{\zeta \in \tilde{I}^{-}(\pi / 4, n-1)} \omega\left(\zeta, I^{-}(\pi / 4, n), B_{n}\right) \\
& \geq \frac{C_{0}}{C} \omega\left(-i, \tilde{T}_{n-1}, \tilde{B}_{n-1}\right) \\
& \geq \frac{C_{0}}{C} \omega\left(-i, T_{n}, B_{n}\right)
\end{aligned}
$$

where $C$ is as in Claim 4.6, and $C_{0}=(1 / A) \omega\left(-i / 2, I^{-}(\pi / 4,0), \mathbb{D} \cap\{\operatorname{Im} z<-1 / 4\}\right)$; here $A$ is the Harnack constant of $\tilde{I}^{-}(\pi / 4, n-1) \cup\left\{-2^{n-1} i\right\}$ in $B_{n}$ (which can be chosen independent of $n$ ). 
Proof of Claim 4.6. To show (4.5), choose $\phi \in C^{\infty}\left(\mathbb{R}^{2}\right)$ such that $\phi \equiv 1$ on $|z| \geq 3 / 4$ and $\phi \equiv 0$ on $|z| \leq 1 / 4$. For $n=1,2,3, \ldots$, let $\phi_{n}(z)=\phi\left(2^{-n} z\right)$. Then, letting $A_{n}=\left\{2^{n-2} \leq|z| \leq 3 \cdot 2^{n-2}\right\}$, we have

$$
\int_{A_{n}} \Delta \phi_{n}(z) d A(z)=\int_{A_{0}} \Delta \phi(z) d A(z) .
$$

Using Green's Identity on (an exhaustion of) $B_{n}(n=1,2,3, \ldots)$ for $G_{n}(z, i)$ and for $\phi_{n}$ we obtain

$$
\int_{A_{n}} G_{n}(z, i) \Delta \phi_{n}(z) d A(z) \geq \omega\left(i, \partial B_{n} \cap\left\{|z| \geq 3 \cdot 2^{n-2}\right\}, B_{n}\right) .
$$

The next lemma is modeled after Lemma 1 of [An79]. We include a proof below, for completeness.

Lemma 4.10. Let $R \geq 4$ and let $v$ be either the Green function for $\Omega \cap\{|z|<R\}$ with pole at $i$, or the harmonic measure of $\{|z|=R\}$ in $\Omega \cap\{|z|<R\}$. Then there exists a constant $C_{1}>1$ independent of $R$ and $\Omega \in \mathcal{S}$, such that

$$
v(z) \leq C_{1} v(|z| i)
$$

for every $z \in \Omega \cap\{2 \leq|z| \leq R\}$.

Using Lemma 4.10 in the case of $v(z)=G_{n}(z, i)$, in equation (4.14), and by Harnack's inequality,

$$
\omega\left(i, I^{+}(\pi / 4, n), B_{n}\right) \leq \omega\left(i, T_{n}, B_{n}\right) \leq C_{2} G_{n}\left(2^{n-1} i, i\right)
$$

for every $n=3,4,5, \ldots$, every $\epsilon>0$, and every $\Omega$ as above.

On the other hand, we note that for all $z$ in $K_{n}=\left\{\left|z-2^{n-1} i\right|=2^{n-2}\right\}$, and all $n \geq 3, G_{n}\left(2^{n-1} i, z\right) \leq C_{3}$, compare with the Green function for the domain $\left\{|z|<2^{n}\right\}$. Moreover, for $z \in K_{n}, \omega\left(z, I^{+}(\pi / 4, n), B_{n}\right) \geq C_{4}$, compare with the corresponding harmonic measure of $I^{+}(\pi / 4, n)$ in the domain $\left\{|z|<2^{n}, \operatorname{Im} z>1 / 4\right\}$. Therefore, by the maximum principle,

$$
\begin{aligned}
\omega\left(i, I^{+}(\pi / 4, n), B_{n}\right) & \geq \omega\left(i, K_{n}, B_{n}\right) \min _{\zeta \in K_{n}} \omega\left(\zeta, I^{+}(\pi / 4, n), B_{n}\right) \\
& \geq \frac{C_{4}}{C_{3}} G_{n}\left(i, 2^{n-1} i\right) .
\end{aligned}
$$

Proof of Lemma 4.10. (See Figure 4.) We extend $v=0$ in the complement of $\Omega$. Then $u(z)=v(\bar{z})-v(z)$ is subharmonic on $\{|z|<R, \operatorname{Im} z>0\}$, and by the maximum principle $v(\bar{z}) \leq v(z)$ for all points in $\{|z|<R, \operatorname{Im} z>0\}$, or what is the same

$$
v(z) \leq v(\bar{z})
$$

for all points in $\{|z|<R, \operatorname{Im} z<0\}$.

Let $C_{0}>1$ be the Harnack constant for the set $\left\{e^{i \theta}: \pi / 8 \leq \theta \leq 3 \pi / 8\right\}$ in the upper half-plane. Then, for all $z$ 's in $\{|z| \leq R / 2, \arg z=\pi / 8\}$,

$$
v(z) \leq C_{0} v\left(e^{i \pi / 4} z\right) .
$$

We claim that (4.16) remains true for $z \in\{R / 2 \leq|z| \leq R, \arg z=\pi / 8\}$. If $v$ is the harmonic measure of $\{|z|=R\}$ in $\Omega \cap\{|z|<R\}$, then the Schwarz reflection principle extends $v$ to a positive harmonic function on the upper half-plane; on the other hand, if $v$ is the Green function for $\Omega \cap\{|z|<R\}$ with pole at $i$, then one uses 


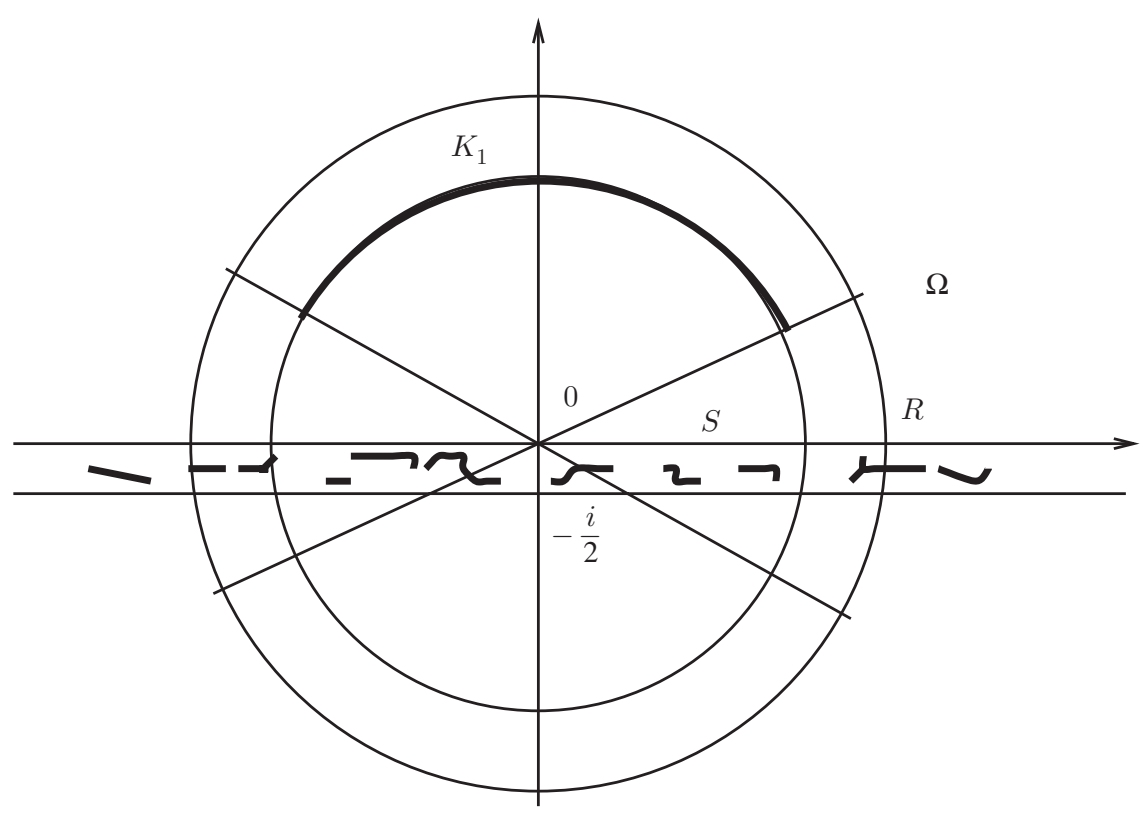

Figure 4. Ancona's Lemma

the boundary Harnack principle for the compact set $\{1 / 2 \leq|z| \leq 1, \pi / 8 \leq \arg z \leq$ $\pi / 4\}$ in the unit disk $\mathbb{D}$, (in this simple case the boundary Harnack principle can also be deduced from Schwarz reflection principle, see for instance Lemma 8.5.1 of AG00 ).

Consider the function $U(z)=v(z)-C_{0} v\left(e^{i \pi / 4} z\right)$, which is subharmonic in the sector $S:=\{|z|<R,|\arg z|<\pi / 8\}$. Note that $U \leq 0$ on $\partial S \cap\{|z|=R\}$ and on $\partial \Omega \cap S$. Also $U \leq 0$ on $\partial S \cap\{\arg z=-\pi / 8\}$ by (4.15), while $U \leq 0$ on $\partial S \cap\{\arg z=\pi / 8\}$ by (4.16). Thus by the maximum principle inequality (4.16) holds for all $z \in S$.

Likewise, we obtain a similar inequality in the sector $S^{\prime}:=\{|z|<R,|\arg z-\pi|<$ $\pi / 8\}$. So if $2<r<R / 2$ is fixed, for all $z$ with $|z|=r$

$$
v(z) \leq C_{0} \sup _{\zeta \in K_{1}} v(\zeta)
$$

where $K_{1}=\{|z|=r,|\arg z-\pi / 2| \leq 3 \pi / 8\}$. Let $C_{1}>1$ be the Harnack constant for the set $\{|z|=1 / 2,|\arg z-\pi / 2| \leq 3 \pi / 8\}$ in $\mathbb{D} \cap\{\operatorname{Im} z>(1 / 2) \sin (\pi / 8)\}$. Then

$$
v(z) \leq C_{0} C_{1} v(r i)
$$

for all $|z|=r$. Hence Lemma 4.10 is proved.

Proof of Claim 4.9. Given $\delta>0$, by harmonic majorization, we can choose $\epsilon$ small enough so that

$$
\sup _{z \in T_{n}} \omega\left(z, L(\epsilon, n+1), B_{n+1}\right) \leq \frac{\delta}{\tau_{0}(1+\delta)}
$$

for $n=1,2,3, \ldots$, where $\tau_{0}$ is given by Corollary 4.7 Also, by letting $\epsilon$ be even smaller we can assume that the claim holds for $n=1,2$ and 3 . We now proceed by 
induction. Assume the assertion true for $n$, we will prove it true for $n+1$ :

$$
\begin{aligned}
\omega\left(i, L(\epsilon, n+1), B_{n+1}\right) & \leq \omega\left(i, T_{n}, B_{n}\right) \sup _{\zeta \in T_{n}} \omega\left(\zeta, L(\epsilon, n+1), B_{n+1}\right) \\
& \leq(1+\delta) \omega\left(i, I(\epsilon, n), B_{n}\right) \frac{\delta}{\tau_{0}(1+\delta)} \\
& \leq \delta \omega\left(i, I(\epsilon, n+1), B_{n+1}\right)
\end{aligned}
$$

where the second inequality follows by the induction hypothesis, and the last inequality follows from Corollary 4.7 This ends the proof of Claim 4.9 .

4.2. Functions of finite order. We show Theorem 4.1 (ii). Note first that $\operatorname{dim} \mathcal{F} \leq 2$. This follows by repeating the proof of Theorem 2 of $[\mathrm{Be} 80$ ] verbatim and using (4.1). (See also Theorem II of Kje51.)

To show that $\operatorname{dim} \mathcal{F} \geq 1$, consider the sequence of harmonic functions

$$
h_{n}(z)=\frac{\omega\left(z, T_{n}, B_{n}\right)}{\omega\left(i, T_{n}, B_{n}\right)}
$$

which are positive, equal to 1 at $i$, and form a normal family. Fix $2 \leq m<n$ and $z \in B_{m}$. Then

$$
\omega\left(z, T_{n}, B_{n}\right) \leq \omega\left(z, T_{m}, B_{m}\right) \sup _{\zeta \in T_{m}} \omega\left(\zeta, T_{n}, B_{n}\right) .
$$

By Lemma 4.10

$$
\sup _{\zeta \in T_{m}} \omega\left(\zeta, T_{n}, B_{n}\right) \leq C_{1} \omega\left(2^{m} i, T_{n}, B_{n}\right)
$$

and by Harnack

$$
\omega\left(2^{m} i, T_{n}, B_{n}\right) \leq 3^{m} \omega\left(i, T_{n}, B_{n}\right)
$$

Thus, for $z \in B_{m}$,

$$
h_{n}(z) \leq 3^{m} C_{1} \omega\left(z, T_{m}, B_{m}\right) .
$$

Hence, the same holds for any normal limit $h$ of the $h_{n}$ 's. So $h$ is a positive harmonic function on $\Omega$, which vanishes on $\partial \Omega$, is equal to 1 at $i$ and is of finite order, i.e. $\operatorname{dim} \mathcal{F} \geq 1$.

4.3. A thickness condition. We show Theorem 4.1 (iii). Let $\mathcal{V}$ be the family of subharmonic functions $v$ on $\Omega$, bounded on $\{\operatorname{Im} z \leq 0\}$, such that for $\zeta \in \partial \Omega$

$$
\limsup _{z \rightarrow \zeta} v(z) \leq 0
$$

and such that,

$$
\limsup _{t \rightarrow+\infty} \frac{\max \{v(z):|z|=t, \operatorname{Im} z>0\}}{t} \leq 1
$$

Then $\mathcal{V}$ is a Perron family, namely, it contains $v_{0}(z)=\max \{0, \operatorname{Im} z\}$, it is closed under maxima, and under Poisson modifications. By Perron's method, it follows that

$$
u(z)=\sup _{v \in \mathcal{V}} v(z)
$$

is either harmonic or identically $+\infty$. We claim the thickness condition (4.2) implies that $u$ is harmonic. 
Let $v$ be a candidate in $\mathcal{V}$. Then $\max _{x \in \mathbb{R}} v(x)<\infty$, and by Phragmen-Lindelöf

$$
v(z) \leq \operatorname{Im} z+\max _{x \in \mathbb{R}} v(x)
$$

for all $z$ with $\operatorname{Im} z>0$. In particular,

$$
\max _{z \in \mathbb{R}+i} v(z) \leq 1+\max _{x \in \mathbb{R}} v(x)
$$

Moreover, since $v$ is bounded for $\operatorname{Im} z<1$, we have

$$
v(z) \leq\left(\max _{w \in \mathbb{R}+i} v(w)\right) \omega(z, \mathbb{R}+i, \Omega) .
$$

Thus, taking the supremum over $z=x \in \mathbb{R}$ in (4.20) and substituting in (4.19), we find that

$$
\max _{z \in \mathbb{R}+i} v(z) \leq 1+\max _{z \in \mathbb{R}+i} v(z) \max _{x \in \mathbb{R}} \omega(x, \mathbb{R}+i, \Omega)
$$

and, by 4.2),

$$
\max _{z \in \mathbb{R}+i} v(z) \leq \frac{1}{1-\max _{x \in \mathbb{R}} \omega(x, \mathbb{R}+i, \Omega)}<\infty .
$$

This shows that $u$ is harmonic on $\Omega$. Note that $u$ is positive since $u(z) \geq v_{0}(z)$, and that $u$ vanishes on $\partial \Omega$, because $\partial \Omega$ is regular. Also, by (4.20) and (4.21),

$$
u(z) \leq \frac{1}{1-\max _{x \in \mathbb{R}} \omega(x, \mathbb{R}+i, \Omega)}<\infty
$$

for all $z$ with $\operatorname{Im} z<1$. Finally, by (4.18) and (4.21), $u$ has finite order. Therefore $u \in \mathcal{F}$. By a similar argument, we construct $\tilde{u} \in \mathcal{F}$, bounded for $\operatorname{Im} z>0$, so that $u$ and $\tilde{u}$ are linearly independent, and $\operatorname{dim} \mathcal{F} \geq 2$.

4.4. The case of translation-invariant domains. Suppose $\Omega$ is a domain such that $\Omega+1=\Omega$. Suppose further that $E=\mathbb{C} \backslash \Omega \subset\{-1 / 2<\operatorname{Im} z<0\}$, that $\operatorname{Cap}(E)>0$, and that $\partial \Omega$ is regular for the Dirichlet problem. We claim that $\mathcal{P}=\mathcal{F}$. Fix $u \in \mathcal{P}$. Note that there exists a simple path $\gamma$ connecting $i$ to $-i$ in $\Omega \cap\{|\operatorname{Im} z|<1\}$. Also let $\gamma_{k}^{+}\left(\gamma_{k}^{-}\right)$be the arc of $\{|z|=|k+i|\}$ connecting $k+i$ to $-k+i$ in the upper (lower) half-plane. So for $k$ large enough, the curve

$$
\Gamma_{k}=(\gamma+k) \cup \gamma_{k}^{+} \cup(\gamma-k) \cup \gamma_{k}^{-}
$$

is a Jordan curve containing $i$ in its interior domain, $D_{k}$.

Consider $k=2^{n}$. Then there is a Harnack constant $C>1$ so that for all $z \in \gamma_{k}^{+}, u(z) \leq C^{n} u(i k)$; for all $z \in \gamma_{k}^{-}, u(z) \leq C^{n} u(k-i)$; and for all $z \in \gamma \pm k$, $u(z) \leq C u( \pm k+i)$. So, we conclude that for all $z \in \Gamma_{k}$,

$$
u(z) \leq C^{n} \frac{u(i)}{\omega\left(i, T_{n}, B_{n}\right)} \leq(2 C)^{n}
$$

where we used (4.12) and then (4.4). Note that for $n$ large enough $\left\{|z| \leq 2^{n-1}\right\} \subset$ $D_{k}$. In particular, this shows that $u$ is of finite order so $u \in \mathcal{F}$. 


\section{LAST REMARK}

We apply the description of the Martin boundary obtained in the previous section to show that whenever the complement of a translation-invariant domain is contained in a strip, then that domain provides a counterexample to the Denjoy-Wolff theorem.

Theorem 5.1. Suppose $\Omega$ is a domain with $\Omega+1=\Omega, E=\mathbb{C} \backslash \Omega \subset\{-1 / 2<$ $\operatorname{Im} z<0\}, \operatorname{Cap}(E)>0$, and such that $\partial \Omega$ is regular for the Dirichlet problem.

(1) Every positive harmonic function on $\Omega$, vanishing on $\partial \Omega$, equal to one at $z_{0} \in \Omega$, is of the form $H=\alpha H^{+}+\beta H^{-}, 0 \leq \alpha, \beta \leq 1, \alpha+\beta=1$, where $H^{+}$is bounded on half-planes $\{\operatorname{Im} z<t\}$, while $H^{-}$is bounded on half-planes $\{\operatorname{Im} z>s\}$.

(2) There exist $w_{1} \neq w_{2} \in \Omega$ such that the sequences $\left\{w_{1}+n\right\}$ and $\left\{w_{2}+n\right\}$ do not have common limit points in the Martin compactification of $\Omega$.

Proof. By parts (iii) and (iv) of Theorem 4.1, it follows that $\operatorname{dim} \mathcal{P}=\operatorname{dim} \mathcal{F}=2$. The boundedness properties of $H^{+}$and $H^{-}$follow from (4.22). This proves part (1).

By Théoreme 2 of [An79], it follows that the sequence

$$
\frac{G(z, i t)}{G(i, i t)}
$$

must converge to a minimal Martin kernel $H$, uniformly on compact subsets of $\Omega$, as $t$ tends to $+\infty$. Thus $H(z)$ is either $H^{+}(z)$ or $H^{-}(z)$. However, by Lemme 1 of [An79], there is an absolute constant $c_{1}>0$ so that,

$$
\frac{G(i R, i s)}{G(i, i s)} \geq\left(1 / c_{1}\right) \frac{G(z, i s)}{G(i, i s)}
$$

for all $s>0$, and for all $|z|=R>0$. So

$$
H(i R) \geq\left(1 / c_{1}\right) H(z)
$$

for all $|z|=R$. Since $c_{1}$ does not depend on $R, H=H^{-}$would imply that $H$ is bounded on $\Omega$, but this is impossible, so $H=H^{+}$. Finally, by Théoreme 1 of An79] we have

$$
\frac{G(i R, i+n)}{G(i n / 2, i+n)} \simeq \frac{G(R i, i n)}{G(i n / 2, i n)}
$$

with a constant $c_{4}$ independent of $R>0$ and $n$. However, by similar arguments as in (3.1) and (3.2), we obtain again that

$$
G(i R, i+n) \simeq[G(R i, i n)]^{2}
$$

with constants independent of $R$ and $n$. So

$$
\frac{G(i R, i+n)}{G(i, i+n)} \simeq\left(\frac{G(R i, i n)}{G(i, i n)}\right)^{2}
$$

and as $n$ tends to $+\infty$ the right-hand side tends to $\left(H^{+}(R i)\right)^{2}$. Hence, one gets different limits by choosing different values of $R$. 


\section{FURTHER RESUlTS AND OPEN PROBLEMS}

In this section, we describe a positive result along these lines and ask some questions.

Question 6.1. Consider all translation-invariant regular domains, i.e. $\Omega+1=\Omega$. We say $\Omega$ is a DW-domain if there is a unique minimal Martin kernel $H$ in the Martin boundary of $\Omega$ so that for every $w \in \Omega$ the orbit $w+n$ tends to $H$ in the Martin topology. Characterize all the DW-domains.

Theorem 5.1 shows that if the complement of $\Omega$ is contained in a horizontal strip, then $\Omega$ is not a DW-domain.

On the other hand, by generalizing a proof given in [ADP-C] for different purposes, we obtain the following result.

Theorem 6.2. Let $\Omega+1=\Omega$, and suppose that

$$
\lim _{R \rightarrow \infty} \sup _{|y|>R} \omega(i y,\{x=1\})=0 .
$$

Then $\Omega$ is a DW-domain.

The proof of Theorem 6.2 is similar to the proof of Theorem 0.4 of ADP-C]. Hence, we will only sketch the main ideas without going into details.

Remark 6.3. Although condition (6.1) is quite general, it is easy to find DWdomains which do not satisfy it. For instance let $\Omega$ be the upper half-plane minus a slanted slit $L=\{x \geq 0, y=-x+1\}$ and also all of its translates by 1 . It is not hard to modify the assumption (6.1) to include these types of examples. However, a more serious example would be given by $\Omega_{0}$ which is the plane minus small disks $D_{n, m}(\epsilon)$ of radius $\epsilon<1 / 4$, centered at the Gaussian integers $n+i m$. This domain is translation invariant; it clearly does not satisfy (6.1). We can show that the orbits $w+n$ converge to a unique Martin kernel $H$. This is the kernel obtained by considering the exponential map $\phi(z)=e^{2 \pi z}$ and the domain $G=\phi\left(\Omega_{0}\right)$. One shows that $G$ has a unique Martin kernel at infinity and then $H$ is the pull-back of that kernel via $\phi$. However, we are not sure whether $H$ is minimal in this case. We leave these questions to further investigations.

Sketch of proof of Theorem [6.2. For every closed subset $E$ of $\{x=0\}$ of positive capacity, consider positive harmonic functions on $\Omega$ of the form

$$
h_{n}(z)=\frac{\omega(z, E+n)}{\omega\left(z_{0}, E+n\right)} .
$$

We study the family $\mathcal{H}(E)$ of all possible normal limits of the $h_{n}$ 's. Namely we show that $\mathcal{H}=\bigcup \mathcal{H}(E)$ contains exactly one function $H$. Moreover, there exists a constant $\rho=\rho(\Omega)>1$ such that

$$
H(z+1)=\rho H(z)
$$

for all $z \in \Omega$. Also, for every $z \in \Omega$, there is a constant $B=B(z)$ such that

$$
\frac{1}{B} \rho^{-n} \leq \omega(z,\{x=n\}) \leq B \rho^{-n} .
$$

The number $\rho(\Omega)$ measures the width of the domain $\Omega$. For instance, if $\Omega$ is the horizontal strip of height $L$, then $\rho(\Omega)=\exp (\pi / L)$. Furthermore, suppose $\Omega_{1}$ and 
$\Omega_{2}$ are two domains as above such that $\Omega_{1} \subset \Omega_{2}$ and $\Omega_{2} \backslash \Omega_{1}$ is a set of positive capacity. Then $\rho\left(\Omega_{1}\right)>\rho\left(\Omega_{2}\right)$.

Consider the family $\mathcal{R}$ of positive harmonic functions $v$ on $\Omega$, which vanish on $\partial \Omega \backslash\{\infty\}$, which are equal to 1 at some base-point $z_{0}$ on $\{x=0\}$, and which have the property that for every constant $a>0$ :

$$
\lim _{z=x+i y \rightarrow \infty, x<a} v(z)=0 .
$$

For a function $v$ in $\mathcal{R}, \max _{x=n} v(x+i y)$ is finite for all $n$ 's. Thus for such functions it makes sense to consider the following quantity:

$$
\beta(v)=\limsup _{n \rightarrow \infty}\left\{\omega\left(z_{0},\{x=n\}\right) \max _{x=n} v(x+i y)\right\} .
$$

Our assumptions (6.1) and $\operatorname{Cap}(\partial \Omega)>0$ imply that $m=\max _{x=0} \omega(z,\{x=1\})<1$. So the conditional probability inequality yields $\omega\left(z_{0},\{x=n\}\right) \leq m^{n}$. The following result provides a dichotomy for the growth of functions in $\mathcal{R}$. It's proof follows the final arguments in the proof of Theorem 4.1 (i).

Proposition 6.4. If $v \in \mathcal{R}$ and $\beta(v)=+\infty$, then for every $S>1$ there is $n_{0}$ such that for $n \geq n_{0}$

$$
\max _{x=n} v(x+i y) \geq S^{n} .
$$

At the risk of introducing too many notations, we will write $\mathcal{R}_{f}$ for the set of functions in $\mathcal{R}$ for which $\beta$ is finite. First notice that the family $\mathcal{H}$ is non-empty by normal families. We show that $\emptyset \neq \mathcal{H} \subset \mathcal{R}_{f}$ along the same lines as Lemma 3.14 of [ADP-C]. Finally, we show that $\mathcal{R}_{f}$ consists of a single function. Equation (6.2) then follows easily, because the function $H(z+1) / H\left(z_{0}+1\right)$ belongs to $\mathcal{R}_{f}$ as well. Hence $H(z)=H(z+1) / H\left(z_{0}+1\right)$, which is (6.2) with $\rho=H\left(z_{0}+1\right)$. It can be shown that $H$ must tend to infinity along the sequence $\left\{z_{0}+n\right\}_{n=0}^{\infty}$, so we deduce that $\rho>1$.

In order to prove that $\mathcal{R}_{f}$ consists of a single function we first prove the following substitute of the Boundary Harnack Principle (see Theorem 3.17 of [ADP-C]).

Proposition 6.5. Suppose $v_{1}$ and $v_{2}$ are functions in $\mathcal{R}_{f}$. Then there is a constant $C>1$ such that

$$
\frac{1}{C} v_{1}(z) \leq v_{2}(z) \leq C v_{1}(z)
$$

for all $z \in \Omega$.

If $\partial \Omega \backslash\{\infty\}$ is smooth enough, e.g. consists of two graphs of Lipschitz functions defined on the whole real axis, then any two positive harmonic functions vanishing on $\partial \Omega \backslash\{\infty\}$ satisfy (6.5) by the Boundary Harnack Principle and by invariance. The main point is to show that this holds without any smoothness requirement. Once Proposition 6.5 is established, the rest of the proof follows a classical argument of Kjelberg (see Kje51 p. 24). In fact, choose two functions $v_{1}$ and $v_{2}$ in $\mathcal{R}_{f}$. Let

$$
m_{n}=\inf _{x=n} \frac{v_{2}(x+i y)}{v_{1}(x+i y)}
$$

Then, $m_{n}$ decreases as $n$ tends to infinity, because we have

$$
v_{2}(x+i y)-m_{n+1} v_{1}(x+i y) \geq 0
$$


for $x=n+1$, hence, by (6.3) and the maximum principle,

$$
v_{2}(z)-m_{n+1} v_{1}(z)>0
$$

for every $z$ in $\Omega \cap\{x<n+1\}$. So, $m_{n} \geq m_{n+1}$. By Proposition6.5. $1 / C \leq m_{n} \leq C$, for some constant $C>1$. So, the limit

$$
1 / C \leq m=\lim _{n \rightarrow \infty} m_{n} \leq C
$$

exists finite and different from zero. Now assume that $v_{2}-m v_{1}$ is not identically zero. Then $v_{2}-m v_{1}$ is a positive harmonic function on $\Omega$. Since both $v_{1}$ and $v_{2}$ are 1 at $z_{0}$, we have $m<1$. Let

$$
w(z)=\frac{v_{2}(z)-m v_{1}(z)}{1-m} .
$$

Then $w \in \mathcal{R}_{f}$. By Proposition 6.5, we have $w(z) / v_{1}(z) \geq 1 / C$ for every $z \in \Omega$ and for some constant $C>1$. However,

$$
\inf _{x=n} \frac{w(z)}{v_{1}(z)}=\frac{m_{n}-m}{1-m} \longrightarrow 0
$$

as $n$ tends to infinity, and this is a contradiction. Therefore, $v_{2} \equiv m v_{1}$. Evaluating at $z_{0}$, we have $m=1$, and $v_{1} \equiv v_{2}$. Thus $\mathcal{R}_{f}$ contains a unique function $H$.

It now follows easily that $H$ belongs to the Martin compactification of $\Omega$ and that given any point $w \in \Omega$ the orbits $w+n$ tend to $H$ in the Martin topology. The last property we need to check is that $H$ is minimal. Assume $v$ is a harmonic function on $\Omega$ and $0<v \leq H$. Then $v / v\left(z_{0}\right)$ belongs to $\mathcal{R}_{f}$. Thus $v=v\left(z_{0}\right) H$, hence $H$ is minimal.

In the course of these proofs we make essential use of the two lemmas below, which we state without proof.

Lemma 6.6. If (6.1) holds, then

$$
\sup _{x=0} \omega(x+i y,\{x=1,|y|>R, d(z, \partial \Omega)<\epsilon\}) \rightarrow 0
$$

as $\epsilon$ tends to 0 and $R$ tends to infinity. Moreover,

$$
\lim _{z \rightarrow \infty, x<0} \omega(z,\{x=1\})=0 .
$$

Lemma 6.7. If $E \subset\{x=0\}$ is as above, there is a constant $D>1$ such that for every $m \geq 1$,

$$
\sup _{x=0} \omega(z, E+m) \leq D \omega\left(z_{0}, E+m\right) .
$$

\section{REFERENCES}

[An79] A. Ancona, Une propriété de la compactification de Martin d'un domaine euclidien, Ann. Inst. Fourier Grenoble, 29, 4 (1979), 71-90. MR 81f:31013

[An84] A. Ancona, Régularité d'accès des bouts, et frontière de Martin d'un domaine euclidien. (French) [Regularity of attainability of ends and Martin boundary of a Euclidean domain] J. Math. Pures Appl. (9) 63 (1984), no. 2, 215-260. MR 86f:31005

[AG00] D. Armitage and S. Gardiner, Classical potential theory. Springer, 2000. MR 2001m:31001

[ADP-C] V. Azarin, D. Drasin, P. Poggi-Corradini, A generalization of $\rho$-trigonometrically convex functions and positive harmonic functions in T-invariant domains, preprint. 
[Ba95] R. Bass, Probabilistic techniques in analysis, Springer-Verlag, New York, 1995. MR 96e:60001

[Be80] M. Benedicks, Positive harmonic functions vanishing on the boundary of certain domains in $\mathbb{R}^{n}$, Ark. Mat. 18 (1980), no. 1, 53-72. MR 82h:31004

[Ca82] L. Carleson, Estimates of harmonic measures. Ann. Acad. Sci. Fenn. Math. 7 (1982), no. 1, 25-32 MR 84i:30032

[Ga89] S. Gardiner, Minimal harmonic functions on Denjoy domains, Proc. Amer. Math. Soc. 107 (1989), no. 4, 963-970. MR 90c:31013

[He41] M. Heins, On the iteration of functions which are analytic and single-valued in a given multiply-connected region, American Journal of Mathematics, Vol. 63, No. 2. (Apr., 1941), 461-480. MR 2:275a

[He88] M. Heins, A theorem of Wolff-Denjoy type, in Complex Analysis, Birkhäuser, (1988), 81-86. MR 90d:30077

[Kje51] B. Kjellberg, On the growth of minimal positive harmonic functions in a plane region, Ark. Mat. 1 (1951), 347-351. MR 12:410f

[Ko88] P. Koosis, The logarithmic integral. I. Cambridge Studies in Advanced Mathematics, 12. Cambridge University Press, 1988. MR 90a:30097

[So94] M. Sodin, An elementary proof of Benedicks' and Carleson's estimates of harmonic measure of linear sets. Proc. Amer. Math. Soc. 121 (1994), no. 4, 1079-1085. MR 94j:30022

Department of Mathematics, Cardwell Hall, Kansas State University, Manhattan, KANSAS 66506

E-mail address: pietro@math.ksu.edu

Current address: Department of Mathematics, East Hall, University of Michigan, Ann Arbor, Michigan 48109

E-mail address: pietropc@umich.edu 